\title{
FAINT GALAXY NUMBER-COUNTS
}

N. METCALFE, T. SHANKS, R. FONG AND J. GARDNER

Physics Depl, Durham University

South Parks Road, Durham DH1 3LE, UK

\author{
AND \\ N. ROCHE \\ Johns Hopkins University \\ Baltimore, USA
}

\section{Introduction}

Observers studying the cosmology and evolutionary history of our Universe through the statistical properties of 'normal' galaxies have four main tools at their disposal. (1) The number-redshift relation. Although a very powerful diagnostic, spectroscopic surveys are currently limited to $B<24^{m}$ and significantly incomplete in the range, $23^{m}<B<24^{m}$. (2) Galaxy numbermagnitude counts. Although by themselves, they cannot constrain models as tightly as spectroscopy, they can be measured $\sim 4^{m}$ fainter, where cosmological effects are expected to be significant. (3) Galaxy colours over a wide wavelength range, which provide additional constraints. (4) The dependence of galaxy clustering with magnitude. $\omega(\theta)$ can be measured to the limit of the counts.

Here we report on the latest Durham count and clustering work.

\section{Durham Galaxy Counts}

At Durham we have been investigating the galaxy number-count and clustering relations for many years. Our original work was done with photographic plates from the 48 in UK Schmidt telescope (limited at $B<21^{m}$ ) (e.g. Stevenson et al. 1986) and from plates taken with the $3.9 \mathrm{~m}$ AngloAustralian telescope $\left(B<23.5^{m}\right)$ (Jones et al. 1991). Since the advent of highly efficient CCD detectors we have concentrated on the deepest possible B-band counts (Metcalfe et al. 1991,1995). 


\subsection{THE DURHAM DEEP FIELD}

Our deepest data all lie on one field, the Durham Deep Field, centred approximately at $00^{h} 20^{m}+00^{\circ} 00^{\prime}(1950)$. We have four major exposures:

(1) a $24 \mathrm{hr}$ B-band exposure with an RCA CCD at the prime focus of the $2.5 \mathrm{~m}$ Isaac Newton Telescope (INT) (Metcalfe et al. 1995), covering an area of $3.3^{\prime} \times 5.7^{\prime}$ (with $0.74^{\prime \prime}$ pixels), with $\sim 1.5^{\prime \prime}$ full-width half maximum (FWHM) seeing. A $3 \sigma$ detection inside twice the seeing FWHM corresponds to a total magnitude of $B=27.0$ for an unresolved object.

(2) a $10 \mathrm{hr}$ B-band exposure with a Tektronix CCD at the auxiliary port of the $4.2 \mathrm{~m}$ William Herschel telescope (WHT) (Metcalfe et al. 1995). This is only a $0.8^{\prime}$ radius circular area ( $0.22^{\prime \prime}$ pixels), centred on the INT field, with a FWHM $\sim 0.9^{\prime \prime}$. With the much improved seeing, the $3 \sigma$ detection limit becomes $B_{\text {total }}=27.6^{m}$.

(3) Our latest work, reported here for the first time, consisting of a $30 \mathrm{hr}$ B-band exposure with a Tektronix CCD at the WHT prime focus. This gives a much bigger field of $7^{\prime} \times 7^{\prime}\left(0.42^{\prime \prime}\right.$ pixel size $)$ which encompasses fields (1) and (2). With a FWHM of $\sim 1.2^{\prime \prime}$, a $3 \sigma$ detection is $B_{\text {total }}=27.9^{m}$

(4) Again reported here for the first time, a $30 \mathrm{hr} \mathrm{K}$-band exposure, covering the $0.8^{\prime}$ radius area of (2), taken with IRCAM3 on the $3.8 \mathrm{~m} \mathrm{UK}$ Infra-Red telescope. The FWHM is $\sim 1.2^{\prime \prime}$ and the $3 \sigma$ limit is $K_{\text {total }} \sim$ $22.5^{m}$.

Combining (1), (2) and (3) creates an ultra-deep field (see fig. 1) equivalent to 50 hours exposure with a $4 \mathrm{~m}$ telescope. The $3 \sigma$ limit on this image, which we believe is the deepest yet, is $B_{\text {total }} \sim 28.3^{m}$. Analysis of this image, and of the K-band data, are still in progress. Ultra-deep $U$ and $R$ exposures on field (3) are scheduled for later in 1995.

\subsection{GALAXY CLUSTERING}

Roche et al. $(1993,1994)$ studied the clustering on the $B \sim 25^{m}$ frames of Metcalfe et al. (1991) and at $B \sim 27^{m}$ on (1) above. In new work, Roche et al. have measured the clustering to $B \sim 26^{m}$ and $R \sim 25^{m}$ over a 400 sq.arcmin. area using $4 \mathrm{hr}$ INT exposures. Work is also underway to determine the clustering at $B \sim 28^{m}$ on our $30 \mathrm{hr}$ WHT exposure.

\section{What do we find?}

The main conclusions which can be drawn from this work are summarised as follows (and see fig. 2):

* The $B<17^{m}$ counts have too steep a slope for the models. As a result, the normalisation for the models is unclear, leading to an uncertainty in the evolution required to fit the fainter counts. 


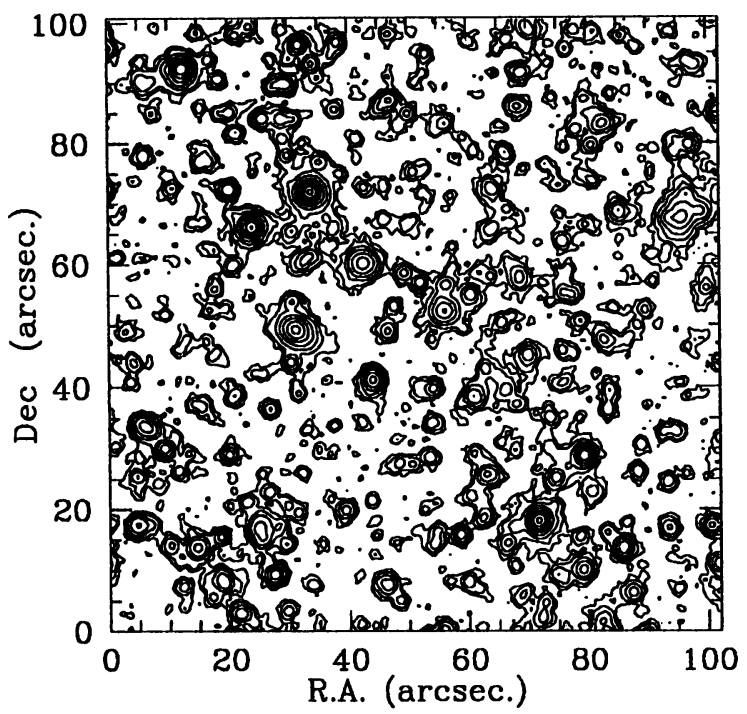

Figure 1. A lightly-smoothed isophotal contour map of our stacked WHT and INT data. The contours are spaced in $1 \mathrm{mag} / \mathrm{sq}$. arcsec apart, with a faintest level of $30 \mathrm{mag} / \mathrm{sq}$. arcsec. Note that there is almost no noise visible in this plot - all the images are real.

* The counts are still rising at $B \sim 28^{m}$, and are uncomfortably high for a closed universe if galaxy numbers are conserved.

* The counts show signs of flattening off at $B>25^{m}$. Such behaviour is indicative of a redshift cut-off or that high redshifts are being reached, where the cosmological volume element starts to increase much less rapidly with redshift. In either case the count slope should then be equal to the faint-end slope of the galaxy luminosity function.

* The B,R,I and K-band counts all seem to have a very similar slope at the limit of observations $(\sim 0.3$ in $\mathrm{d}(\log \mathrm{N}) / \mathrm{dm})$.

* The clustering amplitude is much lower than predicted by non-evolving models. This could be due to a more extended $n(z)$ distribution, lower intrinsic clustering or evolution in the overall clustering strength. There is marginal evidence for a flattening at $B>25^{m}$, consistent with (and for the same reason) as the flattening in the counts.

* Redder galaxies are clustered much more strongly that the blue galaxies at $B>24^{m}$, as expected if they are unevolved early-type galaxies.

\section{References}

Jones, L.R., Fong, R., Shanks, T., Ellis, R.S., Peterson, B.A., 1991, MNRAS, 249, 481

Metcalfe, N., Shanks, T., Fong, R., Jones, L.R., 1991, MNRAS, 249, 498

Metcalfe, N., Shanks, T., Fong, R., Roche, N., 1995, MNRAS, 273, 257

Roche, N., Shanks, T., Metcalfe, N., Fong, R., 1993, MNRAS, 263, 360 

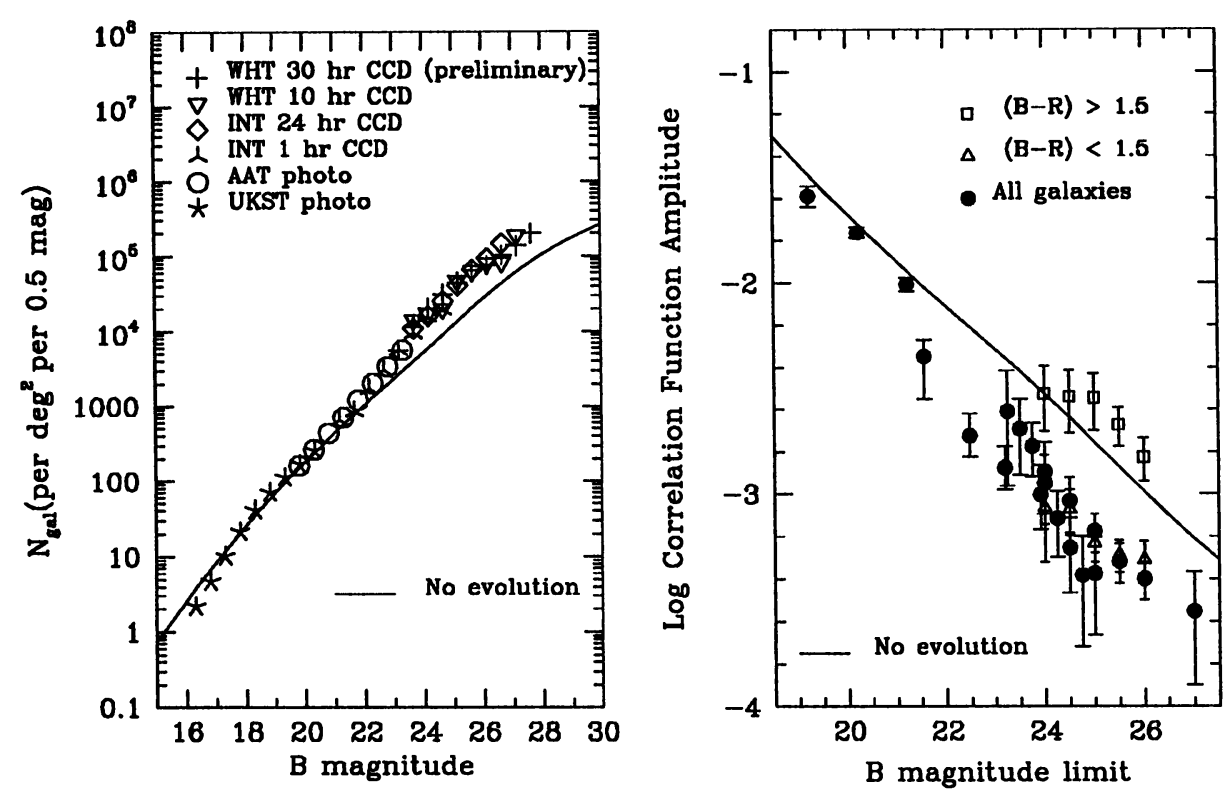

Figure 2. Durham galaxy data: left, B-band galaxy number-counts, corrected for confusion and incompleteness. A low $q_{0}$ non-evolving model prediction is shown. right, B-band clustering amplitude as a function of magnitude, mostly from the same data as used for the counts, again compared with a non-evolving prediction. Our 4 hour INT data is also shown subdivided by colour.

Roche, N., Shanks, T., Metcalfe, N., Fong, R., 1994 in MacGillivray et al., eds, IAU Symp. 161, Astronomy from Wide-Field Imaging. Kluwer, Dordrecht, p. 635

Stevenson, P.R.F., Shanks, T., Fong, R., 1986, in Chiosi, C., Renzini, A., eds, Spectral Evolution of Galaxies. Reidel, Dordrecht, p. 439

\section{Discussion}

WINDHORST: The B-band galaxy counts cannot continue for $B_{J}>28^{m}$ with slope $\alpha>0.4$, or their integrated synchrotron disk-emission at $\mathrm{GHz}$ frequencies would exceed the temperature errors on the cosmic background radiation. So what slope do you infer for $B_{J}>28^{m}$ ?

METCALFE: The count slope has flattened to around 0.3 by the limit of our (confusion corrected) data. In terms of the Schechter luminosity function this would correspond to a faint-end slope of $\sim-1.8$, which if we really are probing high redshifts might imply that the luminosity function was steeper in the past. 